\title{
MOLECULAR ORBITAL STUDY ON INTERFACIAL STRENGTH BETWEEN OXIDE CLUSTER AND POLYIMIDE SUBSTRATE
}

\author{
A. FUJINAMI ${ }^{1}$, S. OGATA ${ }^{1,2}$, Y. SHIBUTANI ${ }^{1,2} \&$ K. YAMAMOTO ${ }^{3}$ \\ ${ }^{1}$ Department of Mechanical Engineering and Systems, ${ }^{2}$ Handai Frontier Research Center, \\ Graduate School of Osaka University, Osaka 565-0871, Japan. \\ ${ }^{3}$ Corporate Manufacturing Innovation Division, Matsushita Electric Industrial Co., Ltd., Osaka 571-8502, Japan.
}

\begin{abstract}
Adsorption processes between silica cluster and poly-( $p$-phenylene pyromellitimide) substrate are investigated by the atomistic calculations combining with the semi-empirical molecular orbital method and the algorisms that can find minimum energy paths of chemical bonding process. Especially, the activation and sorption energies in the reactions are estimated from the optimal reaction path. The Silica thin film-aromatic Polyimide substrate interface is modeled by a silica cluster and polyimide surface which is layered molecular chains with periodic boundary conditions. Conjugate gradient and Quick-Min methods are used to determine atomistic configurations of the system at local minimum and to evaluate physisorption energy. The dimer and the nudged elastic band (NEB) methods, which use only first derivatives of the potential energy, are used in order to search minimum energy paths on the potential energy landscape. The dimer method is started from local minimum on the potential energy landscape and used to search the transition and final states of the system. The NEB method is then carried out to calculate precise path by using final state obtained by the dimer method. From the simulations, we find that the chemical bonding occurs between a silica cluster and polyimide substrate surface, and the sorption energy defined by the sum of physisorption and chemisorption energy are evaluated. Moreover, we discuss the interfacial strength of silica-polyimide substrate using the optimal minimum energy paths.
\end{abstract}

\section{INTRODUCTION}

Understanding the bonding mechanisms of metal or ceramic oxide cluster thin film-aromatic polyimide substrate interface is demanding in its applications for electronic devices because chemical and mechanical stability of such interfaces control authenticity of the devices [1,2]. Therefore, there are many researches have been reported according to interfacial properties of copper-polyimide [2], silicon-polyimide [3] and chrome-polyimide [4]. Since the thickness of component layer in electronic devices is the order of less than submicron, interfacial properties are effected directory by the state of atomic adhesion and interfacial adsorption process. Atomistic simulations such as first 
principle or molecular orbital calculations are effective for investigating the fracture of the interface. Recently, the bond strength of $\mathrm{Fe} / \mathrm{TiX}(\mathrm{X}=\mathrm{C}, \mathrm{N}$ or $\mathrm{O})$ interfaces by using first principle molecular orbital calculations [5], the interfacial strength of substrate-bonded thin metal films by atomistic simulations [6], and the tensile strength and fracture of polar interfaces of the $\{122\} \sum=9$ coincidence tilt grain boundary in cubic $\mathrm{SiC}$ by using the $a b$ initio pseudopotential method based on the local density-functional theory [7] have been reported. In this study, we evaluate the interfacial strength of adsorbed silica cluster on polyimide substrate.

In the adsorption process of the oxide cluster on polyimide surface, there are two kinds of adsorption. One is physisorption (physical adsorption) due to the electrostatic interactions, and which do not involve a significant change in electronic configurations. Another is chemisorption due to nucleation of strong chemical bondings. Generally speaking, chemical bonding at interface is believed to play an important role in adhesion [8]. However, the existence of chemical bonding in the Oxide cluster-Polyimide substrate has not been clarified. In order to clarify whether chemisorption occurs in the adsorption process of Oxide thin film-aromatic Polyimide substrate interface, it is effective to investigate the minimum energy paths (MEPs) of the energy landscape, which is the most probable reaction path. In general case, it is difficult that we guess the MEPs except for very simple systems, since the paths are described in multi-dimensional space. Recently, the dimer method that can search saddle points along the optimal path in the multi-dimensional space with the knowledge of initial states of the system has been proposed by Henkelman and Jónsson [9]. They proposed nudged elastic band (NEB) method that can find the optimal path with the knowledge of initial and final states of the system [10,11]. These methods use only first derivatives of the potential energy.

In the present study, firstly, we investigate interfacial reactions between the silica cluster and polyimide substrate by the semi-empirical molecular orbital calculations and the dimer and NEB methods, and evaluate the activation and sorption energies from the optimal reaction path. Secondary, we propose a method for evaluating ideal interfacial strength of the Silica cluster-Polyimide substrate system.

\section{CALCULATION METODOLOGIES}

In this section, we provide some necessary methodologies for investigating the interfacial reactions of the Silica cluster-Polyimide substrate system and a method for evaluating the strength of the system. In this study, interaction energy and forces of the system are obtained by a semi-empirical molecular orbital method based on PM5 Hamiltonian which is the latest revised version of PM3 [12,13] as coded in a general package MOPAC2002 [14,15]. For the purpose of finding optimal path on the adiabatic potential surface described in the multi-dimensional space, we used the dimer and NEB methods which are described in detail in elsewhere [9-11]. We here describe only a brief overview.

\subsection{The Dimer method}

The dimer method is used to search for final state which is obtained as a result of chemical reaction. It starts from a given configuration $\mathbf{R}_{0}$. Two atomic images (replicas) are displaced a small distance 
$\Delta R$ (in this paper, we set $\Delta R=0.002 \AA$ ) from the common midpoint $\mathbf{R}_{0}$ :

$$
\mathbf{R}_{i}=\mathbf{R}_{0} \pm \Delta R \mathbf{N} \quad(i=1,2)
$$

where $\mathbf{N}$ is a unit vector which defines the dimer orientation.

There are two parts in the algorism to move the dimer. The former is dimer rotation. If the dimer is not exactly located at local minimum, a certain force will act on the images of the dimer. Such forces will pull the dimer to the lowest curvature mode. A modified Newton's method [9] is used to minimize the rotational force which is the difference in the force acting on the images. The latter part of the algorism is translation of dimer. In order to converge to a saddle point, the dimer is moved up the potential surface along the lowest curvature mode, and down the potential in all other curvature mode. This is done by the effective force which brings the dimer to the mid point. The effective force is given by the following equation;

$$
\mathbf{F}_{\text {eff }}= \begin{cases}-\mathbf{F}^{\|} & C>0 \\ \mathbf{F}_{\mathbf{R}}-2 \mathbf{F}^{\|} & C<0\end{cases}
$$

where $\mathbf{F}_{\mathbf{R}}$, is the net translation force acting on the midpoint of the dimer and given as the average of the forces acting on each images. $\mathbf{F}^{\|}$and $C$ are the force acting along the dimer orientation and curvature of the potential, respectively. After each translation, the dimer is reoriented and moved along the path. We adopted an algorism combined with conjugate gradient and Quick-min [9] method in order to minimize the force acting on the midpoint of the dimer. When the dimmer reached to the saddle point, the dimer was located opposite side respect to saddle point along the path. The force acting on the midpoint of the dimer was minimized by using conjugate gradient and Quick-min method, and we eventually obtained the final state of the system.

\subsection{The NEB method}

We adopted the NEB method for searching the optimal path. An elastic band with $N+1$ images described by general coordinate is denoted as $\left[\mathbf{R}_{0}, \mathbf{R}_{1}, \mathbf{R}_{2}, \ldots, \mathbf{R}_{N}\right]$, where $\mathbf{R}_{0}$ and $\mathbf{R}_{N}$ are fixed and given by referring to the local minimum respect to the initial and final states. These initial and final states can be obtained from the dimer method. The $\mathrm{N}-1$ intermediate images are adjusted by the optimization algorism. Atoms between each of images are joined with springs, and the force projection which ensures that the spring forces do not infers with the convergence of elastic band to the MEPs [10]. The total force acting on an image is the sum of the spring force along the local tangent and the true force perpendicular to the local tangent;

$$
\mathbf{F}_{i}=\left.\mathbf{F}_{i}^{s}\right|_{\|}-\left.\nabla E\left(\mathbf{R}_{i}\right)\right|_{\perp} \quad(i=1, N+1)
$$

where $E\left(\mathbf{R}_{i}\right)$ is the total energy which is a function of all the atomic coordinates. The true force is given by;

$$
\left.\nabla E\left(\mathbf{R}_{i}\right)\right|_{\perp}=\nabla E\left(\mathbf{R}_{i}\right)-\nabla E\left(\mathbf{R}_{i}\right) \cdot \hat{u}_{i}
$$


where $\hat{u}_{i}$ is the normalized local tangent at image $i$. The spring force is given by;

$$
\left.\mathbf{F}_{i}^{s}\right|_{\|}=k\left(\left|\mathbf{R}_{i+1}-\mathbf{R}_{i}\right|-\mathbf{R}_{i}-\mathbf{R}_{i-1}\right) \cdot \hat{u}_{i}
$$

where $k$ is the spring constant. In order to move the images according to the force as shown in eqn.(3), we used the optimizing algorism combining with conjugate gradient and Quick-min methods. The climbing image NEB method and variable spring constants are used to obtain the precise MEPs [10].

\subsection{Modeling of the Silica cluster - Polyimide interface}

We adopted rod-like poly-( $p$ - phenylene pyromellitimide) [PMDA-PDA] which have the simplest structure among the polyimides as a polyimide substrate. The optimized atomic structure of PMDA-PDA in one repeating unit is shown in Figure.1. Initial molecular structure of PMDA-PDA was determined by using X-ray structure analysis data reported by Obata et al. [16]. For the purpose of modeling the surface layer, two PMDA-PDA molecular chains are stacked in the $x$ direction as shown in Figure.1. The lower polyimide layer was fixed. We used silica cluster involved two Si and four $\mathrm{O}$ atoms as an adsorbent. The atomic geometries of the $\mathrm{Si}_{2} \mathrm{O}_{4}$ cluster and polyimide substrate were optimized separately. Since there may exist the initial position dependence of the cluster in meta-stable state on the potential surface, we put the optimized $\mathrm{Si}_{2} \mathrm{O}_{4}$ cluster on the 25 different positions in the $y-z$ plane and average distance $\Delta x$ of $4.7 \AA$ above the polyimide surface as shown in Fig.1(b). The region, in which $\mathrm{Si}_{2} \mathrm{O}_{4}$ clusters are put, is one quarter of a repeating unit, since the geometry of the molecular chain is point symmetry. Periodic boundary conditions are set in the $y$ and $z$ directions. Thus, the Silica cluster-Polyimide substrate is modeled as a slab structure. To obtain the atomic geometry in meta-stable state that is the starting geometry of the dimer method, the slab structures were optimized, respectively. The starting geometry for the dimer method is optimized by the algorism combining conjugate gradient and Quick-min method. In this study, the atomic configuration viewer (AtomEye) [17] was used to display atomic configurations.

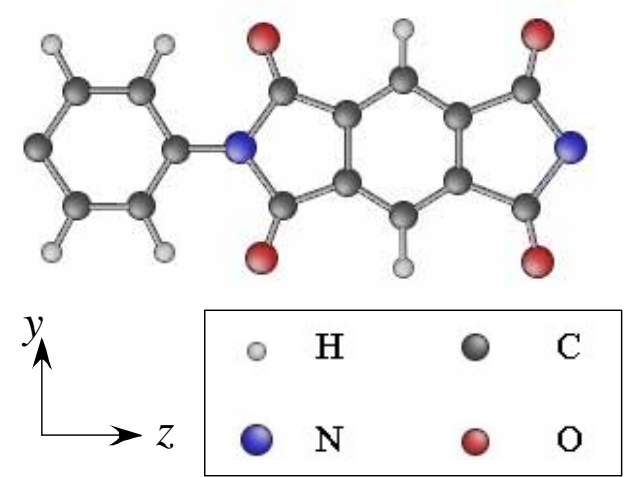

Figure.1 Optimized atomic structure of PMDA-PDA in one repeating unit. 


\subsection{Sorption energy}

In this study, the adsorption process is represented by the relation between the energy of the system and the reaction coordinate. The physisorption energy can be defined as the energy needed to dissociate the cluster from the substrate. We described the physisorption energy by the difference between the energy of meta-stable state $\left(E_{t}\right)$ and sum of the energy of isolated cluster $\left(E_{a}\right)$ and substrate $\left(E_{s}\right)$;

$$
\Delta E_{p}=\left|E_{t}-\left(E_{a}+E_{s}\right)\right| .
$$

The activation energy which is according to rate of reaction is given by;

$$
\Delta E_{a c t}=\left|E_{s a d}-E_{t}\right|
$$

where $E_{\text {sad }}$ is energy of the transition state. The chemisorption energy is also given by;

$$
\Delta E_{c}=\left|E_{t}-E_{f}\right|
$$

where $E_{f}$ is the energy of meta-stable state. Finally, we here evaluated $\Delta E_{p}+\Delta E_{c}$ as the sorption energy.

\subsection{Evaluation of interfacial strength}

The reaction path is given by the following equation;

$$
R_{p}=\sum_{i=0}^{i}\left\|d R_{i}\right\|
$$

where $d R_{i}$ is the displacement between the NEB images and given by;

$$
d R_{i}=\mathbf{R}_{i}-\mathbf{R}_{i-1} .
$$

We assume that the reaction path starts from final state to the saddle point, when the oxide cluster is peeled from the polyimide substrate. The energy-reaction paths curves are fitted by the spline curve as shown in Figure.2(a). Then, the maximum force along MEPs is given by;

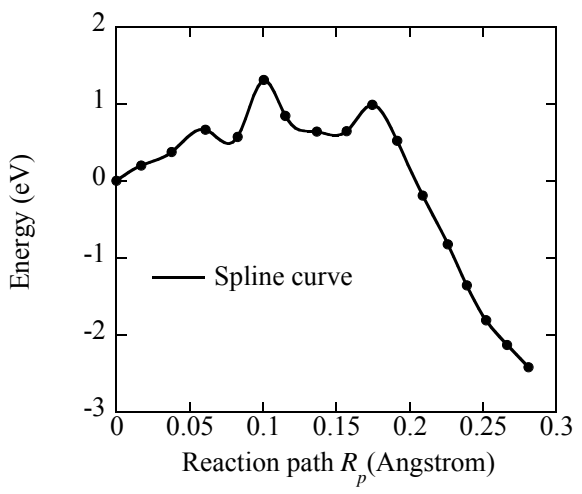

(a)

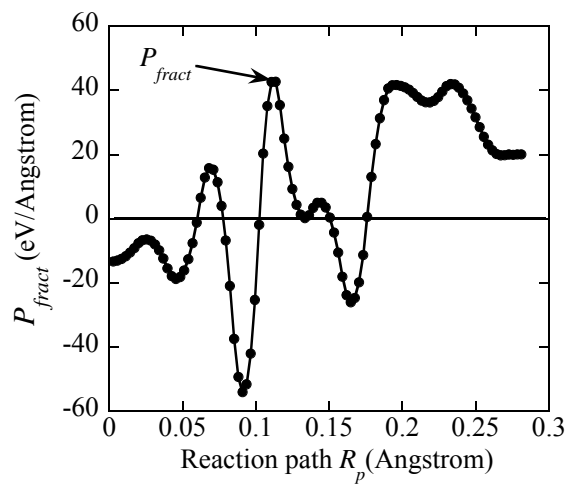

(b)

Figure.2 The evaluation method for fracture load $P_{\text {fract }}$ from the reaction paths of the system. 


$$
\begin{aligned}
\mathbf{F}_{\text {fract }} & =\left(\frac{\partial E}{\partial \mathbf{R}} \cdot \mathbf{n}\right) \mathbf{n}=\left(\frac{\partial E}{\partial \mathbf{R}} \cdot \frac{\left(\mathbf{R}_{i}-\mathbf{R}_{i-1}\right)}{\left\|\mathbf{R}_{i}-\mathbf{R}_{i-1}\right\|}\right) \mathbf{n} \\
& =\frac{\Delta E}{\Delta R_{p}} \mathbf{n}=P_{\text {fract }} \mathbf{n}
\end{aligned}
$$

where $\mathbf{n}$ is normalized unit vector along MEPs. We define the fracture strength as the maximum fracture load $P_{\text {fract }}\{$ Figure.2(b) $\}$ in the eqn.(11) divided by an effective area of one repeating unit of the system $\left\{A_{\text {eff }}\left(90.3-123.8 \AA^{2}\right)\right\}$, and that the fracture strength is positive in the peeling direction of the cluster.

\section{SUMMARY}

In this paper, we descried methods for investigating the interfacial reactions between oxide cluster and polyimide substrate by using semi-empirical molecular orbital method, and the activation and sorption energies from the MEPs. Moreover, we proposed a method for evaluation of the fracture strength of the Oxide cluster-Polyimide interface by using the optimal energy paths. We will give our results and conclusions in the oral presentation.

\section{ACKNOWLEDMENT}

We would like to acknowledge support of this research by Matsushita Electric Industrial Co., Ltd.

\section{REFERENCES}

[1] S.K.Park, Y.H.Kim, J.I.Han, D.G.Moon, W.K.Kim: Thin Solid Films 429(2003), p.231

[2] W.J.Lee, Y.S.Lee, S.K.Rha, Y.J.Lee, K.Y.Lim, Y.D.Chung, C.N.Whang, Appl. Sur. Sci. 205(2003), p. 128

[3] V.Liberman,V.Malba, A.F.Bernhardt, Thin Solid Films, 305(1997), p-26

[4] J.W.Choi, K.E.Lee, T.S.Oh, Mat. Res. Soc. Symp. Vol.629(2000).

[5] M.Mizunio, I.Tanaka, H.Adachi, Acta. Mater. 46(5)(1998), p.1637

[6] Y.L.Shen, J. Mat. Res. 18(10)(2003), p.2281

[7] M.Kohyama, Phys. Rev. B 65(2002), p.184107

[8] M.M.D.Ramos, Vacuum 64 (2002), p255

[9] G.Henkelman, H,Jónsson, J. Chem. Phys.111, 15(1999)7010-7022.

[10] G.Henkelman, J. Chem. Phys. 113, 22(2000)9901-9904.

[11] G. Henkelman, H. Jónsson, J. Chem. Phys. ,113,22(2000)9978-9985.

[12] J.J.P.Stewart, J. Comp. Chem., 10(1989), p.221

[13] J.J.P.Stewart, J. Comp. Chem., 12(1991), p.320

[14] "MOPAC2002", J.J.P. Stewart, Fujitsu Limited, Tokyo, Japan (2001)

[15] J.J.P.Stewart, Quant. Chem. Prog. Exch., 10(1990), p.86

[16] Y.Obata, K.Okumura, S.Kurihara, Macromolecules, 28(1995), p.1547

[17] J.Li, Modelling Simul. Mater. Sci. Eng. 11 (2003), p.173 